Recepción: 20 / 04 / 2017

Aceptación: 20 / 05 / 2017

Publicación: 15 / 09 / 2017
Ciencias de la salud

Artículo Científico

\title{
Marcadores ultrasonograficos en la semana 13 para determinar malformaciones fetales en Guayaquil-Ecuador
}

\section{Ultrasound markers at week 13 to determine fetal malformations in Guayaquil- Ecuador}

\section{Marcadores ultrassonográficos na semana 13 para determinar malformações fetais em Guayaquil-Equador}

\author{
María A. Calero-Zea ${ }^{\text {I }}$ \\ maria.caleroz@ug.edu.ec \\ Blanca E. Andrade-Burgos II \\ blanca.andradeb@ug.edu.ec \\ Ángel A. Sánchez-Alcívar III \\ amando.sancheza@ug.edu.ec
}

\author{
Adriana G. Martínez-Calero ${ }^{{ }^{\mathrm{V}}}$ \\ adrianamartinezcalero@gmail.com \\ Juan J. Calero-Baquerizo v \\ amando.sancheza@ug.edu.ec \\ Julio R. Villacrés-Pastor VI \\ julio.villacresp@ug.edu.ec
}

\section{Correspondencia: maria.caleroz@ug.edu.ec}

I Proyecto de investigación y desarrollo comunitario, APROFE, Universidad de Guayaquil, Guayaquil, Ecuador.

II Especialista en Imagenologia; Doctora en Medicina y Cirugía, Universidad de Guayaquil, Guayaquil, Ecuador.

III Diplomado en Docencia Superior; Especialista en Ginecología y Obstetricia; Diplomado en Docencia Superior; Doctora en Medicina y Cirugía, Universidad de Guayaquil, Guayaquil, Ecuador.

IV Diplomado en Docencia Superior; Diploma Superior en Gestión de Seguridad y Salud en el Trabajo; Diplomado en Docencia Superior; Doctor en Medicina y Cirugía, Universidad de Guayaquil, Guayaquil, Ecuador.

V Médica, Universidad de Guayaquil, Guayaquil, Ecuador.

VI. Médico, Universidad de Guayaquil, Guayaquil, Ecuador. 


\section{Resumen}

Objetivo: Determinar el tipo de malformaciones fetales cromosómicas o no, encontradas en la ciudad de Guayaquil- Ecuador y la edad en las que se presentan en la mujer ecuatoriana. Metodología: El presente estudio es prospectivo y consiste en la utilización de los protocolos de screening del programa del Dr. Nicolaides K. Al realizarlo entre las 13-13,6 semanas se puede adicionar la medición de Arterias Uterinas y la Longitud de Cérvix en un solo examen para lo cual estamos utilizando dos equipos General Electric voluson ProV y Voluson E8. Resultados: El estudio global reporta que fueron atendidas 3169 pacientes en los últimos 13 años, siendo 634 las gestantes vistas en los últimos 18 meses, que corresponde al $20 \%$ del total, representando un aumento del $70 \%$ en relación al año anterior. Dentro de las características demográficas se encontró que la edad materna promedio fue de 29,8 \pm 6.6 años. Conclusiones: La capacitación del profesional debe ser coherente con las expectativas del mismo. También depende de equipos de alta definición de imagen. Además necesita de tiempo para su correcta ejecución.

Palabras clave: Screening; marcadores ultrasonograficos; arterias uterinas; longitud de cérvix. 
María A. Calero-Zea; Blanca E. Andrade-Burgos; Ángel A. Sánchez-Alcívar; Adriana G. Martínez-Calero; Juan J. Calero-Baquerizo; Julio R. Villacrés-Pastor

\section{Abstract}

Objective: To determine the type of chromosomal fetal malformations found in the city of Guayaquil-Ecuador and the age at which they occur in Ecuadorian women. Methodology: The present study is prospective and consists of the use of screening protocols of the program of Dr. Nicolaides K. When performed between 13-13.6 weeks can be added the measurement of Uterine Arteries and Cervical Length in a only test for which we are using two equipment General Electric voluson ProV and Voluson E8. Results: The global study reports that 3169 patients were treated in the last 13 years, 634 of which were seen in the last 18 months, corresponding to $20 \%$ of the total, representing an increase of $70 \%$ in relation to the previous year. Among the demographic characteristics, the average maternal age was found to be $29.8 \pm 6.6$ years. Conclusions: The training of the professional must be consistent with the expectations of the professional. It also depends on high-definition imaging equipment. In addition it needs time for its correct execution.

Keywords: Screening; ultrasound markers; uterine arteries; length of cervix. 


\section{Introducción.}

La difícil situación en la que viven las personas en Ecuador, Sudamérica, por los bajos ingresos económicos per cápita, es agravada entre otras razones, por la contaminación ambiental presente en muchas ciudades ecuatorianas. Esta contaminación, con frecuencia se deriva de esta misma condición de pobreza. ${ }^{1}$

A esto se suman las migraciones hacia las ciudades y el incremento de los Cinturones de Miseria alrededor de las mismas, y en particular alrededor de las ciudades con mayor número de habitantes. ${ }^{1}$

Esta realidad nos ha motivado a trabajar en la presente investigación la misma que deberá terminar en una propuesta viable, que permita contribuir para la disminución de riesgos por contaminantes en la mujer embarazada y en la educación de la misma para la protección de su embarazo, justificando la necesidad de enseñar a nuestra población femenina, la importancia de un estudio ecográfico protocolizado, como screening en el primer trimestre de la gestación para determinar la pesquisa de malformaciones fetales genéticas y congènitas que ya pueden ser detectadas en el primer trimestre. ${ }^{2,3}$

\section{Contribución Potencial del Estudio.}

Ecuador es un país latinoamericano, ubicado geográficamente en la zona tropical y se encuentra atravesado por la Línea Equinoccial. Pese a ser un país con apenas $255.000 \mathrm{~km} 2$ de territorio, posee todos los climas por la presencia de la Cordillera de los Andes, la Amazonía, áreas litorales hacia el Océano Pacífico y la región insular de Galápagos. 4,5 
María A. Calero-Zea; Blanca E. Andrade-Burgos; Ángel A. Sánchez-Alcívar; Adriana G. Martínez-Calero; Juan J. Calero-Baquerizo; Julio R. Villacrés-Pastor

Estas características físicas y climáticas del territorio sumadas a la diversidad de las razas y etnias de los 14 millones de personas que habitan el país, y junto a las crecientes y diversas formas de contaminación presentes, hace necesario el Estudio propuesto. Los resultados del mismo contribuirán al discernimiento de las distintas causas que pueden generan malformaciones fetales en mujeres embarazadas y plantear correctivos, con los consecuentes beneficios para las comunidades y para la Seguridad Social del Estado ecuatoriano. ${ }^{6}$

El objetivo de la presente investigacion es determinar el tipo de malformaciones fetales cromosómicas o no, encontradas en la ciudad de Guayaquil- Ecuador y la edad en las que se presentan en la mujer ecuatoriana.

\section{Metodología.}

Muestra

El presente estudio es prospectivo y consiste en la utilización de los protocolos de screening del programa del Dr. Nicolaides K. Al realizarlo entre las 13-13,6 semanas se puede adicionar la medición de Arterias Uterinas y la Longitud de Cérvix en un solo examen para lo cual estamos utilizando dos equipos General Electric voluson ProV y Voluson E8.

Durante el período 01-01-2016 y el 30-08-2017 se realizaron 634 Screening del Primer Trimestre de los cuales, 456 casos estuvieron entre las 13-13,6 semanas, basándose en la LCR de 65 mm para 13,0 semanas hasta $84 \mathrm{~mm}$ para 13,6 semanas. Los 178 pacientes restantes estuvieron entre las 11 semanas y 12,6 semanas. El grupo de profesionales que realizaron el estudio está acreditado para la realización de los mismos ya que la técnica es fundamental para su confiabilidad.

La técnica para estas mediciones es similar para la TN, $\mathrm{HN}$ y AF, es decir: 
- $\quad$ LCR entre $45-84 \mathrm{~mm}$

- Corte Sagital.

- Posiciòn neutra.

- Magnificación de un $75 \%$

- Habilidad del ecografista.

En esta investigación se realizarán estudios ecográficos a todas las mujeres embarazadas cuya edad gestacional se encuentre entre las 13-13,6 semanas, con la determinación del Cribado Ecográfico del Primer Trimestre:

1. Translucencia Nucal.

2. Ductus Venoso.

3. Hueso Nasal.

4. Ingurgitación Tricuspídea.

5. Ángulo Facial.

6. Longitud de Cérvix.

7. Arteria Uterina.

Especial cuidado tendrá el registro de la edad de la madre. Para los análisis estadísticos posteriores se considerará edades maternas: a) antes de los 18 años, b) entre los 18 y 35 años y, c) después de los 35 años.

Para la realización de este estudio, APROFE cuenta con: 2 Centros Médicos en Guayaquil. 
María A. Calero-Zea; Blanca E. Andrade-Burgos; Ángel A. Sánchez-Alcívar; Adriana G. Martínez-Calero; Juan J. Calero-Baquerizo; Julio R. Villacrés-Pastor

Técnicas de recolección de datos

Para la ejecución sistemática de la obtención de datos, fue necesaria la participación de médicos capacitados en la realización de estos exámenes. Para lograrlo se usò:

1.- La implementación de un área computarizada, donde los profesionales se capacitarán a través de cursos "On Line" impartidos por la FETAL MEDICINE FOUNDATION de Londres.

De esta manera, los médicos encargados de realizar los exámenes, obtendrían sus Certificados de Competencia para la realización de los estudios del Cribado Ecográfico del Primer Trimestre y del estudio Doppler de las Arterias Uterinas.

2.- Se hizo además que los mencionados profesionales se capaciten en L.A.S.U.S. (Latin America School of Ultrasound GE), realizando el "CURSO DE ACTUALIZACION EN ECOCARDIOGRAFIA AVANZADA - LASUS", en la ciudad de Miami Fla., EEUU, para los estudios de Ecocardiografía Fetal, básica y avanzada.

Estos cursos de capacitación son impartidos por el Dr. Fernando Viñals. Otra alternativa que se propone es la de invitar al Dr. Viñals a Ecuador para que los cursos sean impartidos en nuestro medio.

3.- Para la ejecución de la presente investigación, se necesitaron 2 equipos de ultrasonido que atiendan las necesidades tecnológicas que exige el estudio. Una alternativa fue el equipo Voluson E8 con sistema STIC de la marca General Electric, para la realización de los estudios Ecocardiofetales. 
4.- En las mujeres en que se diagnostique alguna patología, se procedió a realizar el respectivo seguimiento con los demás investigadores. El encuestador se trasladò a la vivienda de la paciente, donde procediò a investigar a la madre, a su familia y además, a su entorno. Este seguimiento se realizará hasta el nacimiento del bebé, para confirmar el diagnóstico realizado por Ecografía.

Técnicas y modelos de análisis de Datos.

Esta recolección de información se realizará entre el 01 de enero del 2.016 y el 31 de agosto del 2.017. Para el mismo se escogieron los Centros Médicos de AROFE con atención a más de 5.00 pacientes anuales.

\section{Resultados.}

El estudio global reporta que fueron atendidas 3169 pacientes en los últimos 13 años, siendo 634 las gestantes vistas en los últimos 18 meses, que corresponde al 20\% del total, representando un aumento del $70 \%$ en relación al año anterior. Dentro de las características demográficas se encontró que la edad materna promedio fue de $29,8 \pm 6.6$ años.

De los 456 casos realizados en este período de tiempo, once pacientes tuvieron parámetros elevados en el Screening del Primer Trimestre y corresponden al 2,4\% del total. Cinco pacientes tenían más de 1,6 en la media del IP de Arterias Uterinas y corresponden al 1,09 \% de los 456 pacientes con 13-13,6 semanas y dos pacientes con Longitud de Cérvix menor que $25 \mathrm{~mm}$., y que corresponden al 0,21\% de este grupo poblacional. 
María A. Calero-Zea; Blanca E. Andrade-Burgos; Ángel A. Sánchez-Alcívar; Adriana G. Martínez-Calero; Juan J. Calero-Baquerizo; Julio R. Villacrés-Pastor

La mayoría de las pacientes en la medición de Arterias Uterinas a esta edad gestacional presentaban notch protodiastólico, especialmente las primigestas. Se espera el Doppler de la semana 24 para confirmar su desaparición o persistencia.

En el caso de las dos pacientes con Longitud de Cérvix corto, la primera es una paciente multìpara con antecedente de partos a pretérmino ${ }^{2}$, por lo que realizó cerclaje electivo con $26 \mathrm{~m}$. La segunda paciente, que es primigesta, se realizó cerclaje a la semana 14 con $20 \mathrm{~mm}$.

De las 11 pacientes con paràmetros altos de $\mathrm{TN}$ por encima de $2,9 \mathrm{~mm}$ se realizaron punción de lìquido amniótico siendo 2 Sìndrome de Down( T 21) 0,43\% 1 sìndrome de Eduarws (T 18) 0,21\% 1 Sìndrome de Patau ( T 13).0,21\%

De los 6 pctes con TN anormal DV anormal y FT anormal se detectò una Tetralogìa de Fallot a la semana 24 y $1 \mathrm{CIV}$, en la semanas 22 existen 2 pacientes de el estudio que todavía no alcanzan la semanas 22 para realizarse la ecocardiografía fetal.

Ademàs se pesquizò una arteria umbilical única que corresponde al 0,21\%

No hay que olvidar que en este exàmen no solo se investiga aneuploidìas sino también:

1. Defectos de cara

2. Labio leporino

3. Defectos vertebrales

4. Càmaras cardìacas

5. Cámara gástrica

6. Vejiga

7. Cordòn umbilical 
8. Huesos largos

9. Visualización de manos y pies

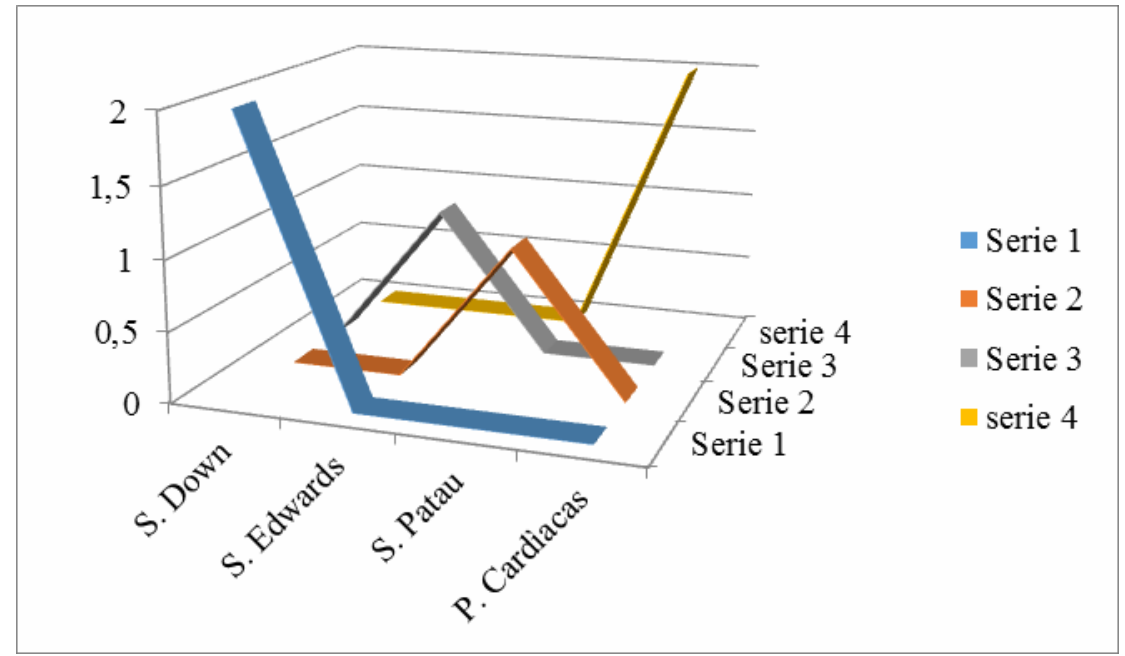

\section{Discusión y conclusiones.}

El estudio del Screening o Cribado del Primer Trimestre, incluyendo todos los parámetros relacionados, es laborioso y operario-dependiente La capacitación del profesional debe ser coherente con las expectativas del mismo. También depende de equipos de alta definición de imagen. Además necesita de tiempo para su correcta ejecución.

Consideramos que es un buen método para poder captar al mayor número de embarazadas posible. Entre el 29-09-2005 y el 02-03-2011 se realizaron 587 Screening y entre el 01-01-2015 y el 01-01-2016 se realizaron 368 Screening. Entre 01-01-2016 y 30-08-2017 se realizaon 634 paciente Un aumento del 70\% de pacientes con relación a años anteriores. 
María A. Calero-Zea; Blanca E. Andrade-Burgos; Ángel A. Sánchez-Alcívar; Adriana G. Martínez-Calero; Juan J. Calero-Baquerizo; Julio R. Villacrés-Pastor

En nuestro país, en vías de desarrollo y con un alto nivel de pobreza, es notable simplificar el número de exámenes, ya que los pacientes tienen poco acceso a la tecnología y los hospitales públicos no logran atender a todos con eficiencia o no cuentan con los equipos y personal capacitado para realizar estos exámenes.

Este examen se podría ofrecer a todas las pacientes, siempre que sea con la información adecuada, para que cada paciente pueda elegir libremente si desea acceder al mismo. Esto debido a que hay pacientes que pueden optar por no conocer cuál es su riesgo, ya que actualmente no existen intervenciones terapéuticas para fetos con aneuploidías, ni existe en nuestro país la opción de un aborto eugenésico.

Todas las pacientes que deseen someterse a este examen deberían tener la alternativa de realizarlo con un especialista con experiencia en esta técnica y, de preferencia con certificaciones. Se evitarían los efectos adversos que se produciría al instaurar esta técnica de manera sistematizada, sin la información y/o experiencia necesaria, y que podrían generar angustia y preocupación innecesaria en las pacientes. Hasta el momento se han añadido màs paràmetros de estudio no hemos centrado exclusivamente en los que incorporan estudio de ultrasonografía sin laboratorio asociado.

Además consideramos que es un estudio simple e importante para detrrminar el bienestar fetal a edades tempranas es necesario concientizar a la ciudadanía sobre su importancia además que es una parñametro imprescindible para el pesquizaje de malformaciones sean estas genèticas o congénitas. 


\section{Bibliografia.}

1. Dezerega V, Sepúlveda W, Schnapps C. Screening en el primer Trimestre de la Gestación. Rev, Med. Clin. Condes 2008;19(3): 165-176.

2. Besio C, Besio M. Consideraciones éticas del uso de la ultrasonografia 11-14 semanas como tamizaje de aneuploidías en la población chilena. Rev. Chil. Obstet. Ginecol. 2009; 74(1):47-51.

3. Cícero S, Curcio P, Papageorghiou A, Sonek J, Nicolaides K. Absence of nasal bone in fetuses with trisomy 21 al 11-14 weeks of gestation. An observational study. Lancet 2001;358: 1665-7.

4. DezeregaV, Sepúlveda W. El examen ultrasonográfico de las 11-14 semanas en: Guzmán E. Rodríguez N. Ruíz M (Eds). Selección de Temas en Gineco- obstetricia, 1 ed. Santiago: Ediciones Publimpacto. 2005; 47-65.

5. Nicolaides K, Falcón O. Características ecográficas de las anomalías cromosómicas. Fetal Medicine Foundation (página web-Internet). Londres 2004: 47-72.

6. Cortéz-Yépez H. Doppler de Arterias Uterinas en el primer trimestre para la detección de trastornos hipertensivos asociados con el embarazo. Estudio de Cohorte. Rev. Colomb. Obstet. 2009; 60(4): 328-33 